\title{
LATTICE-ISOTOPIC ARRANGEMENTS ARE TOPOLOGICALLY ISOMORPHIC
}

\author{
RICHARD RANDELL \\ (Communicated by Frederick R. Cohen)
}

\begin{abstract}
We prove that arrangements which are connected through a smooth family with constant intersection lattice have the same topology.
\end{abstract}

\section{INTRODUCTION}

An arrangement $\mathscr{A}=\left\{H_{1}, H_{2}, \ldots, H_{n}\right\}$ is a finite collection of affine subspaces (of possibly varying dimensions) in $K^{\ell}$, where $K=\mathbb{R}$ or $\mathbb{C}$, or a collection of linear subspaces of a projective space $K \mathrm{P}^{\ell-1}$. The topology of the complement of the union of the $H_{i}$ is of considerable interest. It is well known [4] that if each $H_{i}$ is a hyperplane, one can compute the cohomology ring of the complement of the arrangement from the intersection lattice $L$, and it has recently been shown ([1], [3]) that the lattice determines the homology groups in general. But it is unknown whether $L$ determines the homotopy type of the complement. The main result of this paper shows that if two arrangements are connected by a one-parameter family of arrangements which have the same lattice, the complements are diffeomorphic, hence of the same homotopy type.

\section{PreliminARIES}

We will follow the notation of Orlik's notes [3] throughout; the reader is referred there also for background and further definitions and results. Our main result is true in a number of situations, in particular when the underlying scalars are real or complex. Furthermore, the result holds for arrangements which are linear subspaces of arbitrary dimensions (not just hyperplanes), and for the projective situation as well as the central (all subspaces contain the origin) and noncentral cases in affine space. In order to simplify notation, we will consider arrangements in projective space $\boldsymbol{P}$. Since affine arrangements can be thought of as projective arrangements (with a particular hyperplane at infinity included in the arrangement), the proof we give is general.

Received by the editors September 12, 1988, and in revised form. March 1, 1989.

1980 Mathematics Subject Classification (1985 Revision). Primary 57Q37: Secondary 05B35. 
The lattice of the arrangement has elements corresponding to the intersections of the $H_{i}$, ordered by reverse inclusion. Each $H_{i}$ has a codimension $c_{i}$. We may assume without loss of generality that $H_{i}$ is defined by $c_{i}$ linear equations.

Definition. A smooth 1-parameter family of arrangements $\mathscr{A}$ is a finite collection $\left\{H_{i t}\right\}$ of subspaces for each $t \in \mathbb{R}$ and $i=1,2, \ldots, n$ so that $H_{i t}$ is the locus in $\mathbb{P} \times\{t\} \subset \mathbb{P} \times \mathbf{R}$ of a system of $c_{i}$ equations linear in the variables of $\mathbb{P}$ with coefficients smooth functions of $t$. (That is, the equations have the form

$$
\alpha_{j}(z, t)=\alpha_{j 1}(t) z_{1}+\alpha_{j 2}(t) z_{2}+\cdots+\alpha_{j \ell}(t) z_{\ell},
$$

where the $\alpha_{j m}: \mathbb{R} \rightarrow K$ are smooth functions.) Here and in what follows $j \in J_{i}$, with $\left|J_{i}\right|=c_{i}$.

A one-parameter family gives arrangements $\mathscr{A}_{t}$ for any $t$ in the obvious way.

Definition. Arrangements $\mathscr{A}_{0}=\left\{H_{1}, H_{2}, \ldots, H_{n}\right\}$ and $\mathscr{A}_{1}=\left\{G_{1}, G_{2}, \ldots, G_{n}\right\}$ have the same lattice if for all $I \subset\{1,2, \ldots, n\}$,

$$
\operatorname{dim} \bigcap_{i \in I} H_{i}=\operatorname{dim} \bigcap_{i \in I} G_{i} .
$$

Definition. A 1-parameter family $\mathscr{A}$ is a lattice isotopy provided that for any $t_{1}, t_{2}$, the arrangements $\mathscr{A}_{t_{1}}$ and $\mathscr{A}_{t_{2}}$ have the same lattice.

We do not allow change of field in any of the above definitions. That is, if the original arrangements are over $\mathbb{R}$ (resp. $\mathbb{C}$ ), the isotopy must be defined over $\mathbf{R}$ (resp. $\mathbb{C}$ ). Thus one may apply a complex isotopy to a real arrangement and apply the result below only if one considers the complexification of the real arrangement.

Now denote by $N$ the union of the subspaces and by $M$ the complement of $N$ in $\mathbf{P}$.

\section{RESULTS}

Theorem. If $\mathscr{A}$ is a lattice-isotopy, then $M_{0}$ is diffeomorphic to $M_{1}$ and the pair $\left(\mathbf{P}, N_{0}\right)$ is homeomorphic to $\left(\mathbf{P}, N_{1}\right)$.

Proof. Consider $\mathbb{P} \times \mathbb{P}$ together with the projection map to the second factor. This map is proper since projective space is compact. It is also a submersion. We will describe a Whitney stratification on the domain, constructed from the lattice isotopy, so that the restriction of the projection is a submersion on each stratum. Then the results follow from Thom's first isotopy lemma.

For the convenience of the reader we repeat the relevant definitions. A stratification of a manifold is a partitioning of the manifold into a finite collection of submanifolds $\{U\}$ (called the strata) so that the following frontier condition is satisfied: Whenever $U$ and $V$ are strata with $V \cap \operatorname{cl}(U) \neq \varnothing$, then $V \subset \operatorname{cl}(U)$.

A stratification is called a Whitney stratification if it satisfies Whitney's condition $(b)$ : For all strata $U, V$, with $V \cap \operatorname{cl}(U) \neq \varnothing$, and for all $x \in V$, whenever $x_{i}$ and $y_{i}$ are sequences in $V$ and $U$ respectively with $x_{i} \neq y_{i}$ so 
that $x_{i}$ converges to $x$ and $y_{i}$ converges to $x$, so that the secants $\overline{x_{i} y_{i}}$ converge to $\ell \in \mathbb{R P}^{n-1}$ and so that $T_{y_{i}} U$ converges to $\tau$ in the Grassmannian of dimension $U$ planes in $\mathbb{R}^{n}$, then $\ell \subset \tau$.

We now define a stratification of $\mathbb{P} \times \mathbb{R}$. Any element $X$ of the lattice of $\mathscr{A}$ determines an arrangement $\mathscr{A}^{X}$ in $X$, defined as the intersection of $X$ with all $H_{i}$ of the original arrangement which do not contain $X$. Define $M^{X}$ to be the complement of this new arrangement in $X$. Then one obtains a stratification of $\mathrm{P}$ by taking as strata all $M^{X}$ for $X$ in the lattice, together with a top-dimensional stratum $M$. A stratification of $\mathbf{P} \times \mathbb{R}$ is obtained by taking as strata

$$
V^{X}=\bigcup_{t} M_{t}^{X}
$$

for all $X$ in the lattice. Notice that the closure of the stratum $V^{X}$ is simply the union over $t$ of the subspaces corresponding to the lattice element $X$, and is a manifold of real dimension equal to $\operatorname{dim}(K) \cdot \operatorname{dim}(X)+1$.

To see that $\operatorname{cl}\left(V^{X}\right)$ is a manifold, note that if the lattice element $X$ is the intersection of subspaces $H_{i}$, for $i \in I$, then

$$
\operatorname{cl}\left(V^{X}\right)=\bigcup_{t} \bigcap_{i \in I} H_{i t}=\bigcap_{j \in J_{I}}\left\{\alpha_{j}(z, t)=0\right\}
$$

where $J_{I}$ is the union of the sets $J_{i}$ for $i \in I$.

We define the function $\beta: \mathbb{P} \times \mathbb{R} \rightarrow K^{k}, k=\left|J_{I}\right|$, with coordinate functions $\alpha_{j}, j \in J_{I}$. Since for any fixed $t \operatorname{codim}\left(\bigcap_{i \in I} H_{i t}\right)=c$ is independent of $t$, for all $t$ the matrix $\left(\alpha_{j m}(t)\right), j \in J_{I}, 1 \leq m \leq \ell$, has rank $c$. Choose $c$ rows of this matrix which are independent at $t$, and therefore independent in an interval $U$ about $t$, and use the corresponding functions $\alpha_{j}$ to define a map $\beta^{\prime}: \mathbb{P} \times U \rightarrow K^{c}$. The differential $d \beta^{\prime}$ of $\beta^{\prime}$ is identical to the matrix which has $c$ rows $\left(\alpha_{j}\right)$ and one additional column with entries $\partial \alpha_{j} / \partial t$. Thus $\beta^{\prime}$ has rank $c$ on $\operatorname{cl}\left(V^{X}\right)$. Thus 0 is a regular value of $\beta^{\prime}$, and since $\beta^{\prime-1}(0)=\operatorname{cl}\left(V^{X}\right)$ locally, $\operatorname{cl}\left(V^{X}\right)$ is a manifold.

Observe also that strata $V$ so that $V \subset \operatorname{cl}\left(V^{X}\right)$ are those corresponding to lattice elements greater than or equal to $X$.

It is clear that the projection to the $t$ factor is a submersion when restricted to each stratum.

Finally, we must check that the stratification given is a Whitney stratification. But this holds, because the closure of any stratum is a smooth manifold. Specifically, we have the following

Lemma. Let $x_{i}$ and $y_{i}$ be sequences in $M$ converging to a point $x \in M$ in a smooth manifold $M \subset \mathbb{R}^{k}$ so that $x_{i} \neq y_{i}$ and $\overline{x_{i} y_{i}}$ converges to $\ell$. Then $\ell \subset T_{x} M$.

Proof. This is an application of the mean value theorem. Let $\varphi:(U, 0) \rightarrow$ $(V, x)$ be a parametrization of an open subset $V$ of $M$, defined on an open 
subset $U$ in $\mathbf{R}^{m}$. Let $r_{i}=\varphi^{-1}\left(x_{i}\right), s_{i}=\varphi^{-1}\left(y_{i}\right)$. The mean value theorem gives a point $p_{i}$ on the segment from $r_{i}$ to $s_{i}$ so that $\varphi\left(r_{i}\right)-\varphi\left(s_{i}\right)=d \varphi\left(p_{i}\right)$ $\left(r_{i}-s_{i}\right)^{t}$, the " $t$ " denoting transpose. Normalizing and taking limits gives the result, since $d \varphi\left(p_{i}\right)$ converges to $d \varphi(0)$.

Thus any stratification in which the closure of every stratum is a smooth submanifold is a Whitney stratification. In particular, this is true for the stratification we are using.

The theorem then follows from Thom's first isotopy theorem (proved by Mather [2]). We use the statement from the book of Goresky and MacPherson [1]:

Thom's first isotopy theorem: Let $f: M \rightarrow \mathbb{R}$ be a proper, smooth map which is a submersion on each stratum of a Whitney stratification of $M$. Then there is a stratum-preserving homeomorphism

$$
h: M \rightarrow \mathbf{R} \times\left(f^{-1}(0) \cap M\right)
$$

which is smooth on each stratum and commutes with the projection to $\mathbb{R}$. In particular, the fibers of $f$ are homeomorphic by a stratum-preserving homeomorphism.

Corollary 1. Complements of lattice-isotopic arrangements have the same homotopy type.

Corollary 2. Over $\mathbb{C}$, complements of lattice-isotopic arrangements have isomorphic fundamental groups. Also, $M_{0}$ is a $K(\pi, 1)$ space if and only if $M_{1}$ is.

Corollary 3. Over $\mathbf{R}$, complements of lattice-isotopic arrangements have the same number of components.

Example. Consider the arrangements of Figure 1 as arrangements in $\mathbb{R P}^{2}$ or $\mathbb{C P}^{2}$. Define $Q(x, y, z, t)=\left(x^{2}-z^{2}\right)\left(y^{2}-z^{2}\right)\left(y-x+\left(-2+e^{\pi i t}\right) z\right)$. Over $\mathbb{R}$, $\mathscr{A}_{0}$ is Figure $1 \mathrm{a}$, and $\mathscr{A}_{1}$ is Figure $1 \mathrm{~b}$.

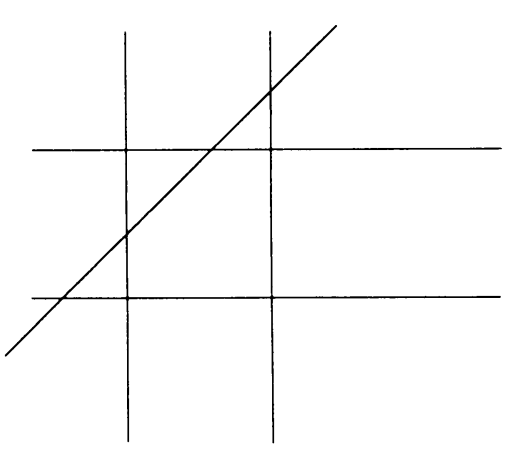

FigURE 1A

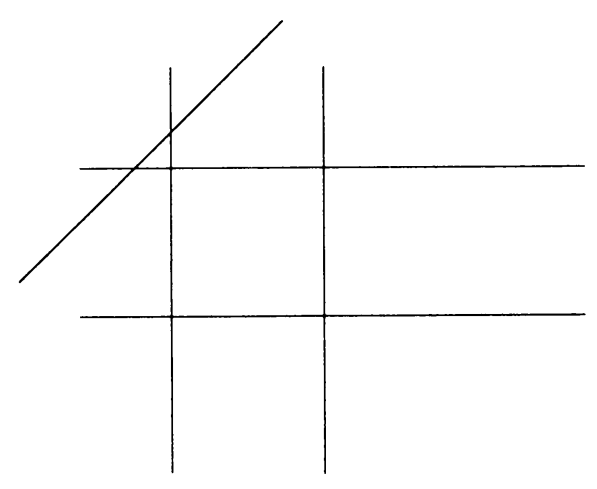

FIGURE 1B 
One may compute directly that $Q$ gives a lattice isotopy, so that $M_{0}$ and $M_{1}$ are diffeomorphic over $\mathbb{C}$. Observe that over $\mathbb{R}$ the "combinatorial" types of the arrangements are different: $\mathscr{A}_{0}$ has a 5-gon, while $\mathscr{A}_{1}$ does not. Thus one might wish to regard these arrangements as different over $\mathbf{R}$ and the same over $\mathbb{C}$.

\section{REFERENCES}

1. M. Goresky and R. MacPherson, Stratified morse theory, Ergeb. Math. Grenzgeb. (3) Vol. 14, Springer, Berlin, 1988.

2. J. Mather, Notes on topological stability, Harvard University, 1970, mimeographed notes.

3. P. Orlik, Introduction to arrangements, Flagstaff CBMS conference, 1988, mimeographed notes.

4. P. Orlik and L. Solomon, Combinatorics and topology of complements of hyperplanes, Invent. Math. 56 (1980), 167-189.

Department of Mathematics, University of Iowa, Iowa City, Iowa 52242 